\title{
Surface Passivation of Germanium Nanowires
}

Hemant Adhikari ${ }^{1}$, Shiyu Sun $^{2}$, Piero Pianetta ${ }^{2}$,Christopher E.D. Chidsey ${ }^{3}$, Paul C McIntyre $^{1}$

${ }^{1}$ Department of Materials Science and Engineering, Stanford University, ${ }^{2}$ Stanford Synchrotron Radiation Laboratory, Stanford University, ${ }^{3}$ Department of Chemistry, Stanford University

\begin{abstract}
The surface of single crystal, cold-wall CVD-grown germanium nanowires was studied by synchrotron radiation photoemission spectroscopy (SR-PES) and also by conventional XPS. The as-grown germanium nanowires seem to be hydrogen terminated. Exposure to laboratory atmosphere leads to germanium oxide growth with oxidation states of $\mathrm{Ge}^{1+}$, $\mathrm{Ge}^{2+}, \mathrm{Ge}^{3+}$, while exposure to UV light leads to a predominance of the $\mathrm{Ge}^{4+}$ oxidation state. Most of the surface oxide could be removed readily by aqueous HF treatment which putatively leaves the nanowire surface hydrogen terminated with limited stability in air. Alternatively, chlorine termination could be achieved by aq. $\mathrm{HCl}$ treatment of the native oxide-coated nanowires. Chlorine termination was found to be relatively more stable than the HF-last hydrogen termination.
\end{abstract}


One-dimensional structures such as nanotubes and nanowires are being actively investigated for various applications in nanotechnology, including nanoelectronics. Silicon and Germanium based semiconductor nanowires are among the more promising of these nanostructures for electronic and other applications because of their compatibility with existing Si CMOS integrated circuit technology. Though, historically, Si replaced Ge in microelectronics largely because of the superior structural and electrical characteristics of the $\mathrm{Si} / \mathrm{SiO}_{2}$ interface, recent work on surface passivation of bulk $\mathrm{Ge}$ crystals and gate dielectric deposition on those surfaces suggests that Ge may again become an important material for high performance transistors ${ }^{1,2}$. In the emerging technology of 3 dimensional (3-D) nanoelectronics, germanium nanowire (GeNW) transistors are very promising components for active device layers above a single crystal silicon substrate because of a) the relatively low growth temperature, well below $400^{\circ} \mathrm{C}$, of these nanowires by catalyzed chemical vapor deposition ${ }^{3}$ and b) the high intrinsic hole and electron mobilities of Ge compared to $\mathrm{Si}^{4}$. The potential of the GeNWs as building blocks for molecular-scale 3-D integrated circuits has been demonstrated by successful fabrication of field-effect transistors (FETs) based on p-type GeNWs with high dielectric constant (high-k) films as gate insulator ${ }^{5}$.

Passivation of the surface of GeNWs before deposition of the dielectric layer is one of the key steps to fabricate a working device. Detailed investigation of the surface chemistry of as-grown and air-exposed GeNWs and exploration of various chemical passivation pathways is valuable for understanding and controlling the behavior of devices made from these GeNWs. 
The GeNWs used in this study were grown using colloidal gold particles (Sigma Aldrich $\mathrm{Co}_{-}$) as the catalyst. Silicon (111) substrates with native oxide were cleaned in Piranha (4 volumes $\mathrm{H}_{2} \mathrm{SO}_{4}: 1$ volume $30 \%$ by mass aqueous $\mathrm{H}_{2} \mathrm{O}_{2}$, heated to $120{ }^{\circ} \mathrm{C}$ ) and SC2 (5 volumes $\mathrm{H}_{2} \mathrm{O}: 1$ volume $37 \%$ by mass aqueous $\mathrm{HCl}: 1$ volume $30 \%$ by mass aqueous $\mathrm{H}_{2} \mathrm{O}_{2}$, heated to $75^{\circ} \mathrm{C}$ ) solutions with thorough water rinses in between. (Aminopropyl)triethoxysilane, APTES, solution was spin coated on these substrates. The APTES monolayer renders the substrates positively charged and allows for the deposition of gold colloids (negatively charged) more efficiently. Subsequently these substrates were dipped in gold colloid solution $($ nominal particle diameter $=10 \mathrm{~nm})$ for $30 \mathrm{~min}$. Nanowire growth was carried out in a cold wall, lamp heated, chemical vapor deposition chamber at $325{ }^{\circ} \mathrm{C}$, with a $\mathrm{GeH}_{4}$ precursor flow of $5 \mathrm{sccm}, \mathrm{H}_{2}$ flow of $550 \mathrm{sccm}$ and a total pressure of 30 torr $\left(\mathrm{P}_{\mathrm{GeH} 4}=0.273\right.$ torr). High resolution TEM reveals that these nanowires are single crystalline with (111) as the predominant growth direction. Photoelectron spectra of these nanowires were carried out using conventional XPS with an $\mathrm{Al} \mathrm{k}_{\alpha}$ source (photon energy $1486.6 \mathrm{eV}$ ) and also using a lower energy synchrotron source (photon energy of $80 \mathrm{eV}$ for Ge $3 \mathrm{~d}$ spectra and $800 \mathrm{eV}$ for survey scans) at the Stanford Synchrotron Radiation Laboratory (SSRL). As the latter methodology gives greater surface sensitivity and allows higher energy resolution, only these results are reported in this letter. Samples described as 'as-grown' were immediately transferred to the photoelectron spectrometer in an argon gas environment. When exposed to ambient atmosphere, samples were shielded with an aluminum foil to avoid, as much as possible, exposure to UV light from fluorescent lamps in the room. 
The as-grown germanium nanowires from our reactor do not show any oxide shifted Ge peaks in XPS (Fig1a). A representative spectrum obtained from a nanowire sample immediately after growth can be fitted extremely well with a Ge3d doublet. (Fig $2 a)^{6,7,8}$. The survey scans performed at $800 \mathrm{eV}$ do not show any evidence of oxygen features (Fig 1b). These data allow us to calculate an upper bound on the average oxygen coverage of these nanowires, which is less than 0.01 monolayers $^{9}$. This suggests that these germanium nanowires are passivated by hydrogen during their growth and this putative hydrogen termination on as-grown wires is stable in argon atmosphere for at least 30 mins. Of the reported XPS studies in literature ${ }^{10,11}$ this is the first report, to our knowledge, to demonstrate oxide free surfaces suggesting completely hydrogen termination of germanium nanowires.

Compared to the well-studied $\mathrm{Si} / \mathrm{SiO}_{\mathrm{x}}$ system, little is known about the mechanism of oxidation of $\mathrm{Ge}$ and the formation of the $\mathrm{Ge} / \mathrm{GeO}_{\mathrm{x}}$ interface on either flat, monolithic surfaces or on nanowires. When exposed to laboratory atmosphere (covered with aluminum foil), the Ge nanowires begin the progressive growth of germanium oxide which can be seen, for example, in the spectrum obtained after varying air exposure times (Fig 1a, 1b). Growth of this surface oxide during short exposures, calculated to be 0.4 and 1.0 monolayer $^{9}$ after 18 min and 33 min of exposure to air, respectively, could not be detected easily using a conventional laboratory XPS system. Different oxides of germanium have been reported to have different properties. For example, GeO desorbs on annealing to $400{ }^{\circ} \mathrm{C}$ or higher, whereas $\mathrm{GeO}_{2}$ has a lower vapor pressure but is water soluble $^{12}$. Hence it is of interest to note the oxidation states of germanium. The Ge $3 \mathrm{~d}$ peaks obtained from nanowires which had been exposed to laboratory atmosphere for all 
exposure times investigated, could be fit as a combination of a bulk $\mathrm{Ge}^{0+}$ substrate feature and three oxide peaks associated with the $\mathrm{Ge}^{1+}, \mathrm{Ge}^{2+}, \mathrm{Ge}^{3+}$ oxidation states with chemical shifts of $0.8 \mathrm{eV}, 1.8 \mathrm{eV}$ and $2.7 \mathrm{eV}$ respectively (Fig $2 \mathrm{~b}$ ). The binding energies of these oxide spectral components are consistent with the literature reports ${ }^{13}$. We did not find the presence of the $\mathrm{Ge}^{4+}$ oxidation state in our nanowires even after long exposures to air at room temperature (e.g. 13 hours in air). While we had taken care to cover these samples with $\mathrm{Al}$ foil to avoid exposure to UV lights (from the atmosphere or from the fluorescent lights in the laboratory), we sought to investigate the effect of controlled UV exposure on nanowire surface passivation. We found that exposing the nanowires to UV lamps for 10 minutes leads to the growth of oxide which had the predominant oxidation state of $\mathrm{Ge}^{4+}$ (Fig 2c). Further studies are in progress to understand the formation and stability of different oxides on exposure to different oxidants including $\mathrm{H}_{2} \mathrm{O}_{2}, \mathrm{O}_{2}, \mathrm{O}$ atoms, and $\mathrm{O}_{3}$ .Controlled oxidation of nanowires may provide a feasible means of passivating the surface during the fabrication process prior to gate dielectric deposition.

Silicon surfaces, etched in aqueous HF have been demonstrated to be extremely clean and are largely hydrogen terminated, leading to remarkably low electronic carrier surface recombination rates ${ }^{14}$. Hydrogen termination of flat Ge (100) after wet chemical treatments was reported only recently. On the basis of thermodynamic considerations, exposure of a Ge surface to aqueous HF should result in F termination, since the Ge-F bond (485 kJ/mol bond enthalpy) is stronger than Ge-H bond $(321 \mathrm{~kJ} / \mathrm{mol})$. However it has been demonstrated that kinetic factors dominate the formation of surface species in the case of bulk (100)Ge substrates ${ }^{15}$, as had been previously established for Si. We have found similar results in the case of Germanium nanowires. As mentioned earlier, when 
exposed to laboratory atmosphere these nanowires grow oxides containing Ge atoms with a range of oxidation states. Most of this native Ge oxide is removed, with the exception of very small residual $\mathrm{Ge}^{1+}$ component (Fig $2 \mathrm{~d}$ ), and putative hydrogen termination is achieved, by immersing the air-exposed nanowires in $2 \%$ aq. HF for 5 min (Fig 3a). Similarly, the UV-exposed GeNWs with a predominantly $\mathrm{Ge}^{4+}$ oxidation state can be hydrogen-terminated by the $2 \%$ aqueous $\mathrm{HF}$ treatment. Comparing the germanium oxide peaks after exposure to air in Fig 1a and Fig 3a, we can see that the stability of the putative hydrogen termination surface obtained by aq. HF treatment (oxide coverage of 1.6 ML after $18 \mathrm{~min}$ in air) is inferior to the one achieved during nanowire growth (oxide coverage of $0.37 \mathrm{ML}$ after $18 \mathrm{~min}$ in air).

The search for an air-stable surface treatment for GeNWs led us to explore the treatment with aqueous $\mathrm{HCl}$. Nanowires exposed to laboratory atmosphere for $13 \mathrm{~h}$ were dipped in $10 \%$ aqueous $\mathrm{HCl}$ for $5 \mathrm{~min}$. This treatment completely removed the oxide shifted germanium features. The narrow scan done at binding energies near $200 \mathrm{eV}$ shows $\mathrm{Cl} 2 \mathrm{p}_{3 / 2}$ and $\mathrm{Cl} 2 \mathrm{p}_{1 / 2}$ peaks at the binding energies of $200 \mathrm{eV}$ and $202 \mathrm{eV}$ respectively, suggesting that after aqueous $\mathrm{HCl}$ treatment the GeNWs are chlorine terminated, instead of the hydride termination obtained after aqueous HF treatment. These germanium peaks can be fitted to a bulk doublet $\left(\mathrm{Ge}^{0+}\right)$, along with a germanium monochloride $\left(\mathrm{Ge}^{1+}\right)$ feature and a germanium dichloride $\left(\mathrm{Ge}^{2+}\right)$ feature shifted from their bulk binding energies by $0.6 \mathrm{eV}$ and $1.2 \mathrm{eV}$, respectively. Although, to our knowledge, the high resolution $\mathrm{Ge} 3 \mathrm{~d}$ spectrum of $\mathrm{Cl}$-terminated $\mathrm{Ge}$ has not been reported in literature for either flat monolithic surfaces or for nanowires, our results are consistent with the Ge2p spectrum obtained from Cl-terminated $\mathrm{Ge}(111)$ by $\mathrm{Lu}^{16}$. A smaller chemical shift in 
binding energy of the $\mathrm{Ge} 3 \mathrm{~d}$ feature for $\mathrm{Cl}$ termination $(0.6 \mathrm{eV})$, as compared to oxygen $(0.8 \mathrm{eV})$, is consistent with the higher electro-negativity of oxygen (3.44 on the Pauling scale) as compared to $\mathrm{Cl}$ (3.16). The chlorine termination obtained by aqueous $\mathrm{HCl}$ treatment is more resistant to oxidation than either the passivation of the as-grown GeNW sample or the passivation obtained by aqueous HF treatment. Even after 77 minutes of exposure to laboratory atmosphere, the oxide coverage for nanowires after aqueous $\mathrm{HCl}$ treatment is calculated to be less than $0.15 \mathrm{ML}$ as compared to untreated GeNWs and HFtreated GeNWs that gave estimated oxide coverages of 1.5 ML and 1.8 ML respectively, after $77 \mathrm{~min}$ exposure to air. The observed robustness of the chlorine-termination can be ascribed to the strength of $\mathrm{Ge}-\mathrm{Cl}$ bond $(431 \mathrm{~kJ} / \mathrm{mol})$, which is larger than that of $\mathrm{Ge}-\mathrm{H}$ bond $(322 \mathrm{~kJ} / \mathrm{mol})$. The reason that $\mathrm{HCl}$ treatment yields $\mathrm{Ge}-\mathrm{Cl}$ bonds while $\mathrm{HF}$ treatment yields putative Ge-H bonds remains unclear. It is worth noting that $\mathrm{HCl}$ treatment does not dissolve oxidized silicon so no direct comparison with silicon is possible.

To summarize, we have studied various chemical passivation routes for cold-wall CVD-grown single crystal germanium nanowires. We have demonstrated that as-grown nanowires are free of oxygen and appear to be completely hydrogen terminated. We have also shown that the chemical states present in surface oxides grown on the_GeNWs are dependent on the oxidizing species. Putative hydrogen termination of GeNWs can be achieved by aqueous $\mathrm{HF}$ treatment whereas $\mathrm{Cl}$-termination can be achieved by aqueous 
$\mathrm{HCl}$ treatment. Chorine termination presents the best passivation we have found for subsequent exposure to room temperature oxidative environments. 


\section{ACKNOWLEDGEMENTS}

We thank Josh Ratchford for helping in the nanowire growth. We are grateful to the members of Nanowire Facility at Stanford Josh Ratchford, Jacob Woodruff and Hemanth Jagannathan for helping maintain the nanowire growth chamber and to Professor Yoshio Nishi for making the growth chamber available. This work is supported by Stanford INMP, DARPA 3D-IC and MARCO MSD center. Portions of this research were carried out at the Stanford Synchrotron Radiation Laboratory, a national user facility operated by Stanford University on behalf of the U.S. Department of Energy, Office of Basic Energy Sciences. 


\section{REFERENCES}

$1 \quad$ H. Kim, C. O. Chui, K. C. Saraswat, and P. C. McIntyre, App. Phy. Let 83 (13), 2647 (2003).

2 C. O. Chui, H. Kim, D. Chi, B. B. Triplett, P. C. McIntyre, and K. C. Saraswat, presented at the IEEE International Electron Devices Meeting (IEDM) 2002, San Francisco, CA, 2002 (unpublished).

D. W. Wang and H. J. Dai, Angewandte Chemie-International Edition 41 (24), 4783 (2002).

S. M. Sze, Physics of Semiconductor devices. (Wiley Interscience, NewYork, 1969).

5 D. W. Wang, Q. Wang, A. Javey, R. Tu, H. J. Dai, H. Kim, P. C. McIntyre, T. Krishnamohan, and K. C. Saraswat, App. Phy. Let 83 (12), 2432 (2003).

Voigt line shapes (convolution of a Gaussian and a Lorentzian) were used during the least-squares fitting, and Shirley background was applied. Other fitting parameters are as follows: $170 \mathrm{meV}$ Lorentzian width, $300 \mathrm{meV}$ Gaussian width, $0.585 \mathrm{eV}$ spin-orbit splitting and 0.667 branching ratio (i.e. statistical value). R. Cao, X. Yang, J. Terry, and P. Pianetta, Phys Rev B 45 (23), 13749 (1992). D. A. Shirley, Phys Rev B 5 (12), 4709 (1972).

R. D. Schnell, F. J. Himpsel, A. Bogen, D. Rieger, and W. Steinmann, Phys. Rev. B, Condens. Matter (USA) 32 (12), 8052 (1985). T. Hanrath and B. A. Korgel, Journal of the American Chemical Society 126, 15466 (2004).

11 D. W. Wang, Y. L. Chang, Q. Wang, J. Cao, D. B. Farmer, R. G. Gordon, and H. J. Dai, Journal of the American Chemical Society 126 (37), 11602 (2004).

12 K. Prabhakaran and T. Ogino, Surface Science 325 (3), 263 (1995).

13 D. Schmeisser, R.D. Schnell, A. Bogen, F.J. Himpsel, D. Rieger, G. Landgren, and J. F. Morar,Surface Science 172, 455 (1986). E. Yablonovitch, D. L. Allara, C. C. Chang, T. Gmitter, and T. B. Bright, Phys. Rev. Lett. (USA) 57 (2), 249 (1986).

15 K. Choi and J. M. Buriak, Langmuir 16, 7737 (2000).

Z. H. Lu, App. Phy. Let 68 (4), 520 (1996). 


\section{FIGURES}
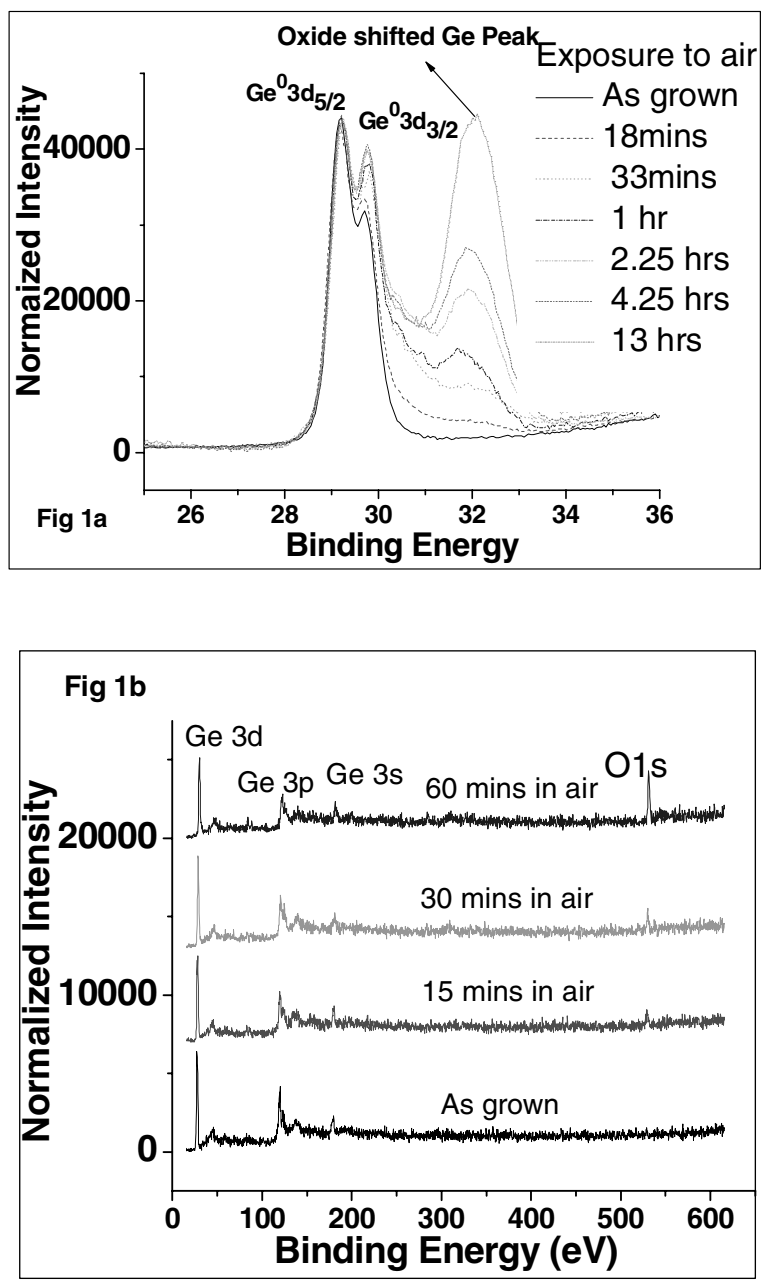

Fig 1. High Resolution Ge 3d (1(a)) and survey scan (1(b)) XPS of as-grown Ge nanowires and the growth of oxide with time. 


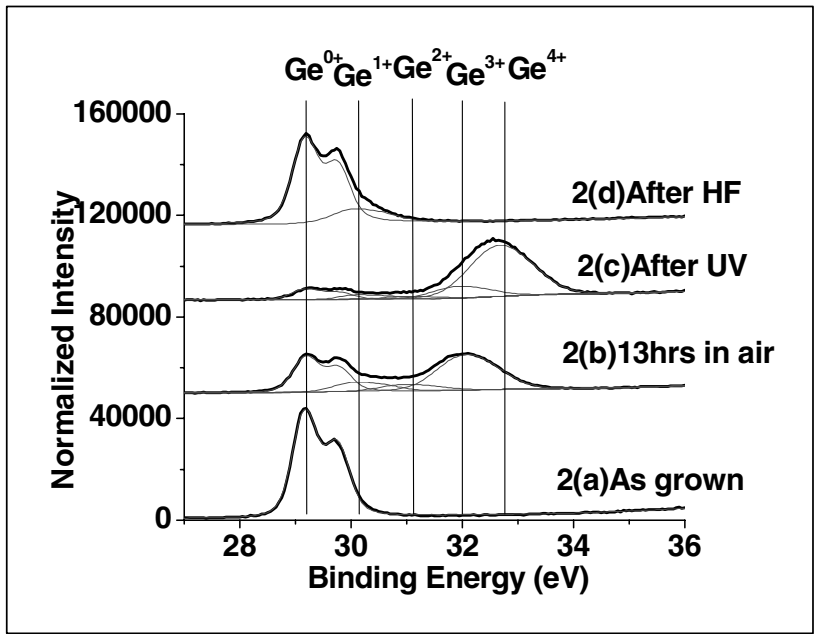

Fig 2. High Resolution XPS of Ge 3d peaks to resolve the presence of various oxidation states. 


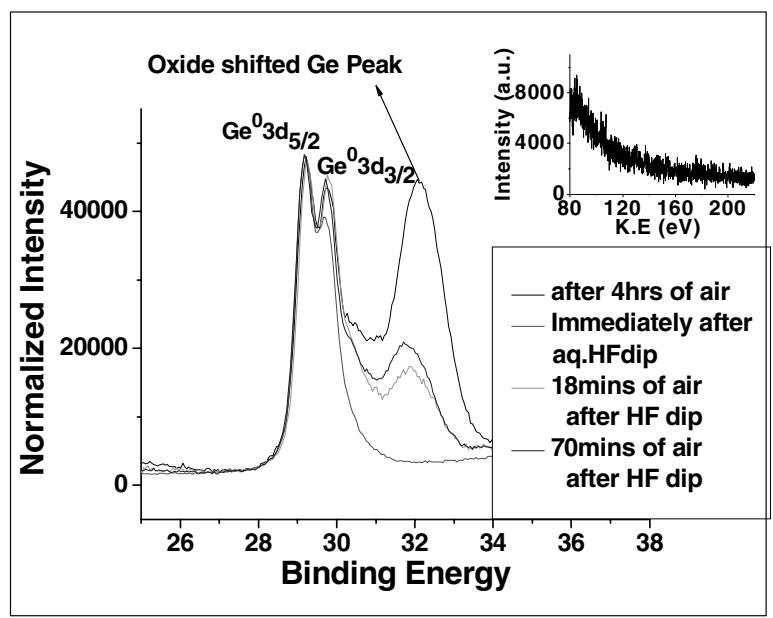

Fig 3.High Resolution Ge 3d XPS of HF-treated GeNWs and the growth of oxide with time. Inset shows the absence of any peak in the range of K.E.s expected for F. 

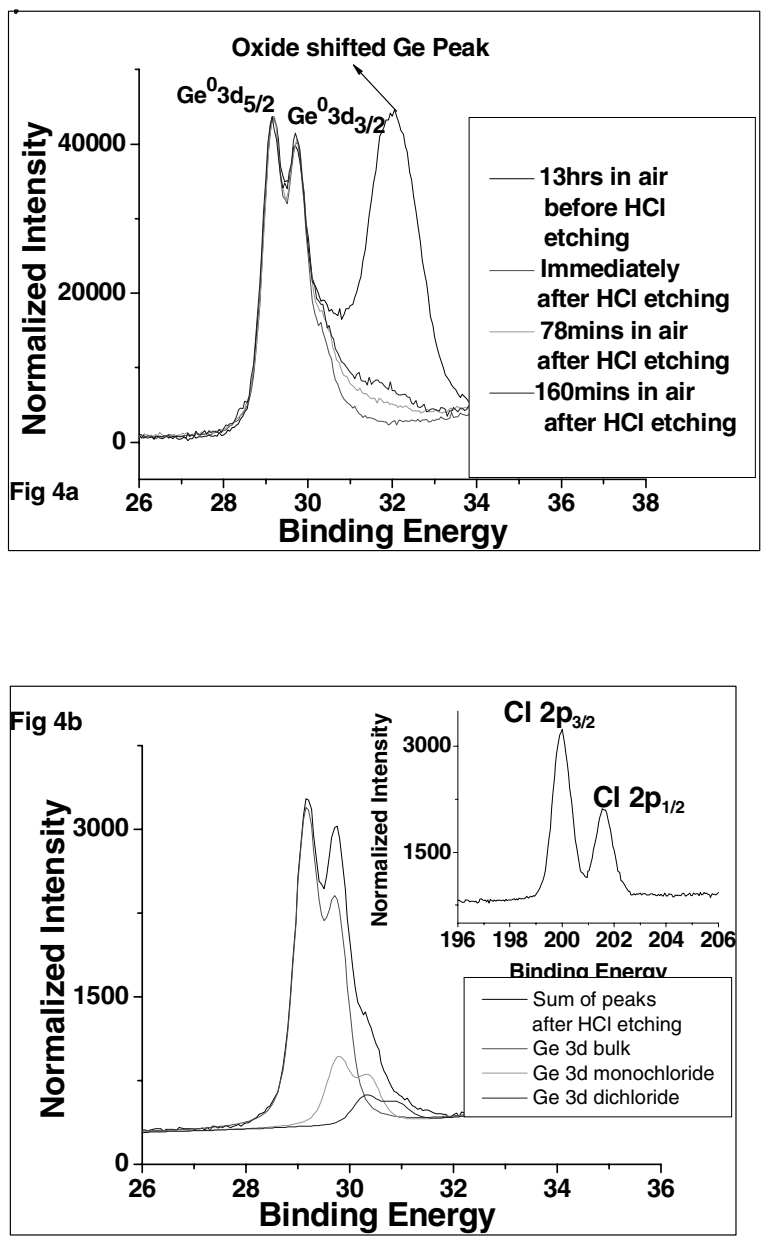

Fig 4a.High Resolution Ge 3d XPS of aq. $\mathrm{HCl}$ treated Ge nanowires and the growth of oxide with time. Fig 4b. Ge 3d peak fitting for Cl-terminated GeNWs immediately after $\mathrm{HCl}$ treatment. Inset shows the presence of $\mathrm{Cl} 2 \mathrm{p}$ peaks. 